\title{
Les exercices de prononciation dans les grammaires de français en Espagne de la première moitié du XIX siècle
}

Pronunciation exercises in French grammars in Spain in the first half of the 19th century

Marc Viémon

\section{CpenEdition}

Journals

\section{Édition électronique}

URL : https://journals.openedition.org/dhfles/6496

DOI : $10.4000 /$ dhfles.6496

ISSN : 2221-4038

\section{Éditeur}

Société Internationale pour l'Histoire du Français Langue Étrangère ou Seconde

Édition imprimée

Date de publication : 1 décembre 2019

Pagination : 313-329

ISBN : 0992-7654

ISSN : 0992-7654

\section{Référence électronique}

Marc Viémon, «Les exercices de prononciation dans les grammaires de français en Espagne de la première moitié du XIX siècle », Documents pour l'histoire du français langue étrangère ou seconde [En ligne], 62-63 | 2019, mis en ligne le 12 avril 2020, consulté le 02 avril 2023. URL : http://

journals.openedition.org/dhfles/6496; DOI : https://doi.org/10.4000/dhfles.6496

Ce document a été généré automatiquement le 2 avril 2023.

Tous droits réservés 


\section{Les exercices de prononciation dans les grammaires de français en Espagne de la première moitié du $\mathrm{XIX}^{\mathrm{e}}$ siècle}

Pronunciation exercises in French grammars in Spain in the first half of the 19th century

Marc Viémon

1 Notre objectif dans cet article est de dresser un panorama de l'évolution pédagogique, au cours de la première moitié du XIXe siècle, des ouvrages grammaticaux de français destinés à un public espagnol en centrant notre attention sur les exercices de prononciation et de lecture. Par « exercice » nous comprenons tout procédé servant à s'exercer, à s'entraîner sur un aspect concret de la langue (Vigner 2016: \$12 ${ }^{1}$ ). L'intégration de ces exercices a contribué à la progressive transformation des grammaires en manuels ${ }^{2}$. Ce processus de transformation est connu sous le nom de manuélisation, "qui concerne la fabrication des grammaires dites "pédagogiques" " (Chiss \& David 2014).

\section{Introduction}

2 L'inclusion d'exercices d'application dans les ouvrages d'apprentissage du français adressés à un public hispanophone n'est pas un phénomène spécifique au XIXe siècle. En effet, ces exercices apparaissent au milieu du XVIIIe siècle chez Antoine Galmace, auteur d'un ouvrage de règles de prononciation (1745) et d'une grammaire proprement dite $(1748)^{3}$. Les exercices en question sont des textes (récit et dialogues) transcrits à l'aide d'un système de prononciation figurée propre.

Cependant plus de la moitié des ouvrages qui comportent des exercices au XVIII ${ }^{\mathrm{e}}$ siècle ne sont pas des grammaires, mais des ouvrages réduits contenant uniquement des 
règles de prononciation. En effet, si nous prenons en compte les œuvres d'apprentissage du français destinées aux Espagnols ${ }^{4}$ et publiées de 1745 à $1799^{5}$, nous en dénombrons 9 sur 19, soit $47 \%$, qui incluent des exercices pour pratiquer la prononciation par l'intermédiaire de la lecture et de la répétition de listes ${ }^{6}$. Cinq de ces ouvrages s'intéressent exclusivement à l'enseignement de la prononciation et de la lecture: ce sont ceux de Galmace (1745), Soldevila (1767), Tallés (1773), González Cañaveras (1781) et Pelleport (f. XVIII'). D'un autre côté, les grammaires proprement dites qui offrent des exercices à leurs usagers sont celles de Grimarest (1747), Galmace (1748), Roca y María (1750) et Chantreau (1781).

4 Tout comme Galmace, Grimarest propose à ses lecteurs une histoire transcrite. Cependant ces deux auteurs sont les seuls à fournir aux usagers ce type d'exercice. D'autres, comme Soldevila (1767) et González Cañaveras (1781), incluent également des textes: il s'agit de prières, non transcrites et sans réelles indications d'utilisation; cependant, les ouvrages étaient uniquement destinés à l'apprentissage de la prononciation ou de la lecture ${ }^{7}$, ce qui ne laisse aucun doute sur la fonction des textes choisis par les deux auteurs ${ }^{8}$. Chantreau (1781), pour sa part, utilise la prononciation figurée dans ses explications sur la prononciation du français, mais ne transcrit pas non plus les textes qu'il insère à la fin de son supplément; des textes (« Anecdotes, bonsmots, faits historiques ») destinés, selon l'auteur, à "s'exercer à la lecture et à la traduction» $(1781: 290)$. Le dernier à proposer un exercice de lecture, non transcrit mais plus élaboré9 , est Tallés (1773).

5 Dans une perspective plus mécaniste, les autres exercices de prononciation se présentent sous forme de listes de difficultés particulières que le lecteur doit répéter afin d'acquérir certains automatismes, chez Roca y María (1750), Tallés (1773), Chantreau (1781) et Pelleport (f. XVIII) ; Chantreau, auteur chez lequel les listes sont les plus élaborées et se présentent parfois sous la forme de paires minimales (ante litteram), propose également un résumé de toutes les règles sur les voyelles, « diphtongues » et consonnes.

6 Finalement, signalons le cas particulier de González Cañaveras (1781) qui propose aux usagers de son ouvrage une espèce de syllabaire ${ }^{10}$ (3-36), sans fournir d'indications particulières de son utilisation, mais également deux chapitres intitulés «Exercicio de las reglas expresadas" (81-94) et "Exercicio de estas reglas en las excepciones» (100-105). Ce sont des listes d'exemples transcrits qui visent à illustrer certaines règles théoriques précédemment exposées. Le terme «Exercicio» n'a pas été choisi par l'auteur au hasard. Mais, là non plus, on ne trouve aucune indication sur le mode d'emploi de ces listes. Il faut donc supposer que l'élève devait les lire sous l'oreille attentive du maître.

7 Sachant qu'avant 1745 aucun ouvrage d'enseignement du français aux hispanophones n'incluait d'exercice, la seconde moitié du XVIII e siècle marque le point de départ d'une pédagogisation progressive des ouvrages grammaticaux de français en Espagne qui, comme nous allons le voir, va s'amplifier dans la première moitié du XIX ${ }^{\mathrm{e}}$ siècle.

8 Nous n'avons malheureusement pas eu accès à la totalité des ouvrages grammaticaux de français publiés à cette époque et adressés aux Espagnols mais nous avons tout de même pu en repérer une vingtaine incluant des indications de prononciation ${ }^{11}$. Disons d'ores et déjà que treize de ces ouvrages proposaient explicitement aux usagers des exercices de prononciation, ce qui représente $65 \%$ du corpus, soit une augmentation importante par rapport à la deuxième moitié du siècle précédent. Par ailleurs, alors 
qu'au XVIII siècle nous ne recensions que quatre grammaires dotées d'exercices - les autres ouvrages étant des recueils de règles de prononciation -, au XIX ${ }^{\mathrm{e}}$ siècle les treize œuvres concernées par l'inclusion d'exercices sont toutes des grammaires. L'évolution pédagogique de ce type d'ouvrage n'en est que plus significative.

9 Avant de voir en détail les ouvrages proposant des exercices de prononciation, nous allons commenter brièvement les grammaires des auteurs n'ayant pas participé à cette évolution pédagogique.

\section{Les ouvrages sans exercices explicitement destinés à la pratique de la lecture/prononciation}

10 Nous recensons sept auteurs de la première moitié du XIXe siècle ne proposant à leurs lecteurs qu'une présentation classique de la valeur des lettres de manière graphophonétique dans leur chapitre de prononciation sans fournir aucun exercice d'application. Chez eux, la grammaire reste un ouvrage purement théorique et la pratique de la prononciation ne semble pas revêtir d'importance. Pour résoudre les difficultés de prononciation, on invite souvent le lecteur à écouter ceux qui parlent "purement et de manière cultivée " (Salvà $1847: 3$ ). Il s'agit des auteurs suivants : Novella (1813), l'auteur anonyme de Gramática filosófica y literaria de la lengua francesa (1816), Tramarría (1829), Fournier (1841), Anglada (1844), Salvà (1847) et Lobé (1850).

11 Le cas de Tramarría est à considérer à part puisque sa grammaire est une réédition de l'Arte de Chantreau publié en 1781. Seulement, ce continuateur supprime les textes originaux ${ }^{12}$, en ajoute un autre appelé « Description de la Bétique par Fénélon » mais ne précise pas comment il faut en faire usage. Ainsi, parmi ceux que nous avons consultés, Tramarría est le rééditeur de Chantreau le moins explicite en termes d'exercices d'application de la théorie phonétique.

\section{Vers une pédagogisation des grammaires : I'inclusion d'exercices explicitement destinés à l'exercice de la lecture et de la prononciation}

\section{Des textes de lecture sans transcription : Chantreau et ses continuateurs}

12 Dans la quatrième édition de sa grammaire, publiée en 1804, Chantreau conserve les textes d'application de la première édition, c'est-à-dire une série d'" Anecdotes, bonsmots, faits historiques, etc., où l'on pourra, en s'amusant, s'exercer à la lecture et à la traduction » (1804:290). À ces 36 textes courts s'ajoutent des « Apophtegmes » (302) et un " Précis historique de l'Espagne » (302). On doit supposer que ces textes ont la même utilité que les anecdotes. Parmi les continuateurs de Chantreau, Dupuy (1836) et Puigarri (1841) conservent exactement la même structure; Alemany (1826) et Bordas (1842), en revanche, la modifient.

13 Alemany, conserve les anecdotes et la référence explicite au fait qu'elles sont un exercice pour lire et traduire ( Anéctodas y agudezas para ejercitarse en la lectura y la traducción»), mais supprime les autres textes présents chez Chantreau et en ajoute 
deux autres : «Cuadro físico de España » et «Cuadro histórico de España » (différents de ceux du «Précis »). Bordas, pour sa part, supprime tous les textes du supplément mais aux pages 14 et 15 de son ouvrage, c'est-à-dire au sein de la partie de prononciation, il propose au lecteur une section pratique intitulée "Práctica de lectura ", sans indications d'utilisation, malheureusement. Cependant il faut signaler que l'exercice en question, certainement dirigé en classe par le professeur, présente une progression dans la difficulté : il est question de lire tout d'abord des mots isolés tous des adjectifs - puis des syntagmes et enfin des phrases. Bordas est le seul auteur de notre corpus mais également des auteurs du XVIII ${ }^{\mathrm{e}}$ siècle à proposer un tel exercice.

Les rééditions de l'Arte de Chantreau sont en grande partie responsables de l'augmentation importante du nombre de grammaires plus pédagogiques au XIXe siècle. Mais tout ne se résume pas à Chantreau: certains auteurs proposent des ouvrages originaux qui s'inscrivent également dans la tendance à la manuélisation.

Beauchemin (1840), tout d'abord, fournit, à l'instar de Chantreau, de nombreuses explications théoriques, particulièrement intéressantes, dans l'introduction de son ouvrage et expose distinctement ses orientations méthodologiques, qu'il résume de façon suivante : peu de règles et beaucoup de pratique.

Concernant la prononciation, il se déclare ennemi de la prononciation figurée et affirme que « la lecture ou l'application immédiate des règles est, sans aucun doute, la meilleure façon de fixer le son dans l'oreille du disciple " (1840:4). Seulement, les textes d'application ne suivent pas immédiatement les règles de prononciation chez cet auteur $^{13}$. Les textes se trouvent en réalité à la fin de l'ouvrage : c'est un recueil de morceaux choisis de littérature, qui s'étend sur près de cent pages (id. : 239-333) visant à « s'exercer à la lecture et à la traduction ».

Grimaud de Velaunde (1826), pour sa part, précise dans son introduction que l'apprenant de français a besoin de «livres élémentaires» (1726: V) pour se perfectionner dans la langue, incluant ainsi à la fin de son ouvrage un Abrégé des Aventures de Télémaque (139 pages) pour le style « élevé » et, un Précis des Aventures de Robinson Crusoe (126 pages) pour le style « familier ». L'auteur indique également dans son introduction qu' «avant que les élèves commencent à lire [...] les professeurs doivent leur faire bien prononcer les sons multipliés et les voix les plus difficiles de la langue française» (id.: VII). Pour ce faire il inclut un syllabaire ${ }^{14}$, sur lequel nous reviendrons.

\section{Des textes avec transcription et numérotés : Galmace et ses épigones}

Les auteurs de la seconde moitié du XIX ${ }^{e}$ siècle ayant inclus dans leurs ouvrages des textes ou des exercices de lecture transcrits se situent dans le sillage de Galmace, dont nous avons rappelé le rôle de précurseur au début de cet article. En effet, c'est le premier auteur à avoir inclus dans ses ouvrages grammaticaux des textes de lecture transcrits à l'aide d'une prononciation figurée propre.

19 Sánchez Ribera (1721:1), dans son ouvrage, considère que la partie la plus difficile de la langue française est la prononciation, ou plutôt sa lecture, du fait de la non correspondance entre l'oral et l'écrit et affirme également que l'« exercice » de la lecture est nécessaire pour fixer dans la mémoire les règles apprises. C'est la raison 
pour laquelle il fait suivre la partie dédiée à la prononciation par un «Exercice de lecture en prose» (id.: 61-80) et d'un autre en vers (id.: 81-91). Le premier est un extrait de l'Abrégé de l'Histoire Romaine de Goldsmith ; le deuxième est composé de deux poèmes, l'un de Boileau et l'autre de Rousseau. Ces trois textes sont transcrits à l'aide d'une prononciation figurée, mais contrairement à Galmace et à Grimarest, la transcription ne se fait pas dans une colonne parallèle mais de façon interlinéaire, ce qui représente une nouveauté.

20 Les exemples fournis à chaque item décrit dans la partie de prononciation sont également transcrits et c'est là que le lecteur doit se reporter pour interpréter les symboles utilisés par l'auteur dans la transcription ${ }^{15}$, ce qui compliquait sans doute la lecture ; il en était de même chez Galmace. Chantreau, en revanche, avait pris la peine de rassembler en une courte liste les symboles de son système de figuration des lettres/ sons.

21 Rodríguez de Chauveau (1837), lui, est un auteur particulier dans ce groupe car les exercices de lecture qu'il propose ne sont pas totalement transcrits : il ne signale que les difficultés vues dans chaque leçon, de façon interlinéaire également.

Dès le titre de sa grammaire, cet auteur annonce clairement son contenu pratique et déclare que chacune des leçons est associée à un exercice d'application, ce qui est totalement inédit chez les grammairiens de français ayant adressé leur ouvrage à des hispanophones. Cette déclaration de principes est confirmée par l'auteur dans le prologue: ayant constaté que l'élève se décourage facilement lorsqu'il se trouve confronté à une infinité de règles et de difficultés, il préconise de réaliser un exercice après chaque règle (ou groupe de règles) $(1837: 4-5): 1$. alphabet et voyelles " simples »; 2. voyelles " composées »; 3 . voyelles nasales; 4. signes orthographiques (pour travailler l'élision) ; 5 . consonnes ${ }^{16}$.

23 La partie théorique de la grammaire est assez sommaire : dans le cas de la première leçon des explications de prononciation, par exemple, il n'aborde que le $\mathrm{E}$ et les voyelles longues et brèves, sur l'étendue de quatre pages, pas plus ${ }^{17}$. Les exercices de lecture correspondants occupent autant d'espace que les règles : ils ciblent les contenus abordés et sont placés à la fin de la leçon dans le souci de les mettre immédiatement en pratique. On se trouve donc face à un ouvrage qui se situe dans le courant théoricopratique mais qui présente la nouveauté de fournir aux usagers les exercices d'application de manière progressive et immédiate, alors que les auteurs précédents proposaient des textes une fois assimilée toute la théorie. Mais le pas suivant est franchi par Pirrefer.

24 Cet auteur prône l'apprentissage par la pratique et non pas par les règles et justifie sa posture méthodologique en pointant du doigt le peu de réussite des méthodes traditionnelles $(1847: 2)$. À ce sujet, il rappelle au lecteur une série de maximes tirées d'auteurs renommés défendant l'apprentissage par l'usage, maximes qui ne sont pas sans rappeler celles que cite Rovira dans le prologue de sa méthode Jacotot adressée aux Espagnols (1835 : VII-XII).

Suivant ses préceptes, Pirrefer modifie l'ordre habituel des méthodes de langue théorico-pratiques et commence directement avec une première partie praticothéorique de 47 pages intitulée «Lectura ». Elle se compose de treize leçons. Les onze premières commencent par un texte qui se présente sous la forme de trois colonnes (français-transcription-traduction) et également, disposé en dessous, le même 
fragment sans prononciation figurée et avec la traduction espagnole interlinéaire. Ce n'est qu'après le texte que l'auteur fournit des explications théoriques.

Les textes correspondent d'abord à des leçons de prononciation (1. alphabet; 2. voyelles «simples»; 3 . voyelles « composées »; 4 . voyelles nasales ; 5 . consonnes ; 6 . valeurs de $\mathrm{S} ; 7 . \mathrm{H}$ « aspiré ») et ciblent les points abordés dans chacune d'entre elles : dans la leçon sept, par exemple, le texte associé contient les mots suivants «homme, hasarder, hasarde, hardi, haute, hêtre, harpe » (1847: 18), qui servent à préparer les règles données postérieurement par l'auteur.

À partir de la leçon huit jusqu'à la onze, Pirrefer fournit des textes à lire et ce sont des notes de bas de page qui fournissent les indications théoriques nécessaires. Les leçons douze et treize sont uniquement théoriques.

Autre particularité de Pirrefer, qu'il partage avec Chantreau : il fournit au lecteur des indications en cinq points sur l'utilisation de son ouvrage dans une section nommée «Ejercicios» (id.: 5). Les deux premiers pas concernent la lecture correcte des textes dont il fournit la transcription : il faut d'abord lire la colonne transcrite puis essayer de lire le texte original de la même manière. Les points trois, quatre et cinq concernent la traduction de chaque texte.

À côté de Sánchez Ribera, Rodríguez de Chauveau et Pifferer, qui ont inclus des textes transcrits dans leurs ouvrages, nous pouvons ranger deux autres auteurs: Bergnes de las Casas (1845) et Cornellas (1850). Ils proposent également des textes d'application à leurs lecteurs mais avec une innovation d'un point de vue formel. En effet, au lieu de transcrire directement les mots à l'aide d'un système de prononciation figurée, ils choisissent de numéroter les difficultés; chaque numéro renvoie à une règle explicative.

Bergnes de las Casas inclut deux exercices de lecture placés après la partie de prononciation. Le premier est une liste de mots, chacun visant une difficulté particulière du vocalisme français, toujours d'un point de vue graphophonétique, et un numéro entre parenthèses placé après chaque mot renvoie à une note en bas de page où la difficulté en question est expliquée. Cette liste s'intitule "Práctica de lectura » (1745 : 26). Quant au deuxième exercice, il s'agit d'un texte précédé du titre «Lectura seguida » (id.: 26-27) dans lequel le lecteur trouve également des numéros, placés audessus des mots cette fois, et qui renvoient aux pages 22 et 23 de la grammaire, où l'auteur expose un résumé des règles; chaque item correspond à un numéro, 33 en tout. Le lecteur peut alors consulter, pour chaque difficulté, la règle correspondante.

Cornellas propose aux lecteurs un système similaire à celui que nous venons de commenter. Après les explications théoriques, dont chacune correspond à un numéro il y en a $134-$-, l'auteur inclut à la page 20 un texte d'application à lire intitulé « Modelo de análisis para la pronunciación, en que se citan las reglas principales». Le texte est très court (onze lignes) et il n'y a aucune indication pédagogique visant à éclairer le lecteur dans la réalisation de l'exercice de lecture à part le titre qui le précède. Le titre du texte se réfère à ce que, au-dessus de chaque mot il y a un numéro qui renvoie à une règle énoncée dans la théorie et à laquelle le lecteur doit se rapporter pour réaliser la prononciation correcte.

Delaborde (1855) et Mendizábal (1860) ${ }^{18}$ suivront l'exemple de Cornellas et Bergnes de las Casas et incluront également dans leurs ouvrages des textes de lecture annotés. 


\section{Les exercices de répétition}

parties qui tentent de fournir aux usagers un panel de combinaisons de lettres puis d'exemples d'applications le plus exhaustif possible. L'auteur commence par diverses listes de syllabes classées selon leur composition: articulations simples "directes", tout d'abord, dans lequel nous trouvons les combinaisons du type consonne + voyelle, puis, tout de suite, articulations simple "inversées ", où l'ordre des syllabes est voyelle + consonne. Cela continue avec des articulations doubles, c'est-à-dire consonne + consonne + voyelle, puis les syllabes que l'auteur appelle «composées»: consonne + voyelle + consonne. Finalement, le lecteur trouve des syllabes de trois consonnes suivies de voyelle. veut travailler : voyelles, consonnes, longueur, nombre de syllabes et $\mathrm{H}$ " aspiré ». Le syllabaire de Grimaud de Velaunde est donc très complet et unique au sein de notre corpus. 
d'une liste de syllabes du type consonne + voyelle, suivant l'ordre alphabétique : «Ba, be, bi, bo, bu, ca, ce, ci, etc. ». Les seules combinaisons de lettres suivies de voyelle qu'il recense sont les suivantes : $\mathrm{CH}, \mathrm{GE}, \mathrm{PH}, \mathrm{QU}, \mathrm{RH}, \mathrm{TH}, \mathrm{SC}, \mathrm{SCR}, \mathrm{SP}$, ST et STR. Il n'offre pas non plus d'indications au lecteur en ce qui concerne l'utilisation du syllabaire.

\section{En guise de conclusion}

Dans l'histoire de l'enseignement des langues, il est rare de trouver des évolutions méthodologiques brutales, des coupures en quelque sorte; au contraire, il existe généralement une certaine continuité et les véritables "innovations" sont peu nombreuses. C'est aussi le cas dans le domaine plus particulier de l'histoire de l'enseignement de la prononciation du français aux hispanophones.

41 Cependant, comme nous l'annoncions au début de l'article, la proportion des ouvrages incluant des exercices d'application, que ce soit des textes de lecture ou des listes de mots ou de syllabes à répéter, augmente de façon assez considérable entre la deuxième moitié du XVIII siècle et la première du XIX ${ }^{\mathrm{e}}$, ce qui est caractéristique, selon nous, de la manuélisation progressive des grammaires de français pour les étrangers: non seulement on commence à laisser plus de place à la pratique au sein même des ouvrages, mais nous assistons également à un renversement - isolé il est vrai - de l'ordre traditionnel théorie-pratique chez Pirrefer qui demandait à ses élèves de commencer par lire la transcription puis le texte original, avant de passer aux règles.

En ce qui concerne les types d'exercices, la transcription de textes, utilisée par Galmace et Grimarest au XVIII ${ }^{e}$, ne connaît pas un réel succès et n'est pratiquée que par trois auteurs, entre autres Pirrefer; en revanche un nouveau procédé de référencement des sons, ou phonèmes dans la terminologie actuelle, voit le jour. Il s'agit de la numérotation des difficultés graphophonétiques et du renvoi, par l'intermédiaire de ces numéros, aux règles de prononciation auxquelles le lecteur devra se rapporter.

Il s'agirait maintenant de compléter cette étude par la consultation du reste des ouvrages publiés à la même époque et pousser le travail d'analyse jusqu'à la fin du XIX ${ }^{\mathrm{e}}$ siècle afin de compléter le panorama que nous avons tenté d'offrir dans cet article.

\section{BIBLIOGRAPHIE}

\section{Sources primaires}

S. n. (1816). Gramática filosófica y literaria de la lengua francesa ó arte de hablar, escribir y traducir el francés correctamente. Bordeaux : Pierre Beaume.

ALEMANY, Lorenzo (1826). Gramática francesa titulada el nuevo Chantreau. Madrid : Aguado. ANGLADA, Francisco (1844). Gramática de la lengua francesa para uso de los españoles ò sea tratado completo de las diferencias gramaticales de dicha lengua comparada con la española. Barcelone : Joaquin Verdaguer. 
BEAUCHEMIN, E. Hippolyte de (1840). Elementos de la lengua francesa ó método práctico para aprender este idioma. Paris : Adolphe Bihourd.

BERGNES DE LAS CASAS, Antonio (1845). Novísimo Chantreau ó gramática francesa. Barcelone : Juan Oliveres.

BORDAS, Luis (1842). Compendio de la Gramática francesa de D. Pedro Nicolas Chantreau ó elementos de gramática francesa dispuesto para el uso de la juventud. Barcelone : Imprenta de Manuel Saurí.

ChantReAu, Pierre-Nicolas (1781). Arte de hablar bien francés, ó Gramatica completa. Madrid : Antonio de Sancha.

CHANTREAU, Pierre-Nicolas (1804). Arte de hablar bien francés ó gramática completa dividida en tres partes. Madrid : Sancha.

CORNELLAS, Clemente (1850) [1845]. Gramática francesa, teórico-práctica, para el uso de los españoles. Logroño : Domingo Ruiz.

DELABORDE, R. E. L. (1855). Novísimo arte teórico, práctico, analítico y sintético de lengua francesa.

Madrid : J. de M. González.

DUPUY, Paul (1836). Arte de hablar bien francés ó gramática completa dividida en tres partes. Barcelone : Francisco Oliva.

FOURNIER, Gustave (1841). Gramática francesa adoptada en el Colegio de Humanidades. Madrid :

Salvador Albert.

GALAVOTTI, Guillermo Luis (1839). Arte de hablar y escribir en francés correctamente ó nueva y completa gramática francesa. Barcelone : Imprenta de Brusi.

GALMACE, Antoine (1745). Adiciones a la Gramatica Francesa, que compuso el R. P.Nuñez. Madrid : s. n.

GALMACE, Antoine (1748). Llave nueva, y universal, para aprender con brevedad, y perfeccion la lengua Francesa. Madrid : Gabriel Ramírez.

GONZÁLEZ CAÑAVERAS, Juan Antonio (1781). Cartilla Francesa ó Combinaciones generales para la lectura. Cadix : Luis de Luque y Leyva.

GRIMAREST, Jean-Henri Le Gallois de (1747). Gramatica Francesa con un nuevo Methodo para aprender a pronunciar. Pampelune : Herederos de Martínez.

GRIMAUD DE VELAUNDE, Francisco (1826). Método práctico para aprender los elementos de la lengua francesa. Madrid : Repullés.

LOBÉ, Carlos (1850). Curso de temas en castellano que comprende toda la sintacsis del idioma francés. Cadix : Imprenta de la Casa de Misericordia.

MENDIZABAL, Joaquín (1860). Gramática francesa. $2^{\mathrm{e}}$ éd., Saragosse : Vicente Andrés.

NOVELLA, Pablo Antonio (1813). Nueva gramática de la lengua francesa y castellana, avec un abrégé de la grammaire espagnole. Alicante : Imprenta de España.

PELLEPORT, Dionisio de (f. XVIII ${ }^{\mathrm{e}}$ ). Reglas para pronunciar, y leer con inteligencia la lengua francesa.

Madrid : s. n.

PIRREFER, Francisco (1847). El idioma francés puesto al alcance de todos, ò método natural para aprender francés de un modo fácil y agradable sin cansar la memoria. Madrid: Despacho de Libros de la Calle Espoz y Mina. 
PUIGARRI, Pierre (1841). El novísimo Chantreau ó Arte de hablar bien francés. Perpignan : Jean-Baptiste Alzine.

RoCA Y MARÍA, Sébastien (1750). Arte Francès, ò Nuevo Methodo Facilissimo, para Leer, Hablar, y Escrivir Francès, conforme à lo mas moderno. Barcelone : Francisco Surià.

RODRÍGUEZ DE CHAUVEAU, Miguel Francisco (1837). Nueva gramática francesa práctico-elemental, dividida en 30 lecciones, cada una de ellas corroborada con un ejercicio. Madrid : Yenes.

ROVIRA, Miguel (1835). Tratado completo de la enseñanza universal, ó método de Jacotot. Barcelone : Herederos de Roca.

SALVÀ, Vicente (1847). Gramática para los españoles que desean aprender la lengua francesa sin olvidar la propiedad y el giro de la suya. Paris : Vicente Salvà.

SÁNCHEZ RIBERA, Juan (1721). Gramática francesa de Lhomond enteramente refundida por Carlos Constante Letellier, Profesor de Bellas Letras, acomodada al uso de los españoles, y enriquecida con un tratado completo de pronunciación y con otras diferentes adiciones útiles. Madrid : José del Collado.

SOLDEVILA, Francisco (1767). Rudimentos breves y claros para aprender a leer, y escribir en francés sin socorro de maestro. Madrid : Antonio Muñoz del Valle.

TALLÉs, Juan Magín (1773). Rudimentos de la pronunciacion Francesa, para el uso de los Caballeros del Real Seminario de Nobles de esta Corte. Madrid : Antonio Mayoral.

TRAMARRÍA, Francisco de (1829). Gramática francesa para uso de los españoles. Madrid : Moreno.

\section{Sources secondaires}

CHISS, Jean-Louis \& DAVID, Jacques (2014). « Les grammaires de référence dans la francophonie : contextualisations et variations ». Langue française 181, 79-95. En ligne : [https://www.cairn.info/ revue-langue-francaise-2014-1-page-79.htm] (consulté le 05 mai 2019).

FERNÁNDEZ FRAILE, María Eugenia (2002). « L'enseignement de la prononciation du français en Espagne au XIX ${ }^{\mathrm{e}}$ siècle ». Documents pour l'histoire du français langue étrangère ou seconde 28, vol. 2. En ligne : [http://journals.openedition.org/dhfles/2633] (consulté le 24 janvier 2019).

GARCíA BASCUÑANA, Juan Francisco (coord.) (2017). Diccionario de historia de la enseñanza del francés en España (siglos XVI-XX) (DHEFE). En ligne : [http://www.grelinap.recerca.urv.ca t/projectes/ diccionario-historia-ensenanza-frances-espana/] (consulté le 24 janvier 2019).

LÉPINETTE, Brigitte (2012). Un demi-siècle de grammaire pour l'enseignement du français en Espagne (1800-1850) : Contexte - Paratexte - Textes. Étude d'Historiographie linguistique. Valence : Universidad de Valencia.

VIÉMON, Marc (2016a). L'apprentissage de la prononciation française par les Espagnols aux XVI ${ }^{e}, \mathrm{XVII}^{e}$ et XVIII ${ }^{e}$ siècles. Thèse de doctorat, Université de Séville (715 p.).

VIÉMON, Marc (2016b). « Apprendre à prononcer le français en Espagne après Galmace (1745) : continuité et nouvelles tendances pédagogiques », Documents pour l'histoire du français langue étrangère ou seconde 57. En ligne : [http://journals.openedition.org/dhfles/4415] (consulté le 24 janvier 2019).

VIGNER, Gérard (2016). « L'exercice ». Carnets Deuxième série - 8. Revue électronique d'Études françaises. A.P.E.F. En ligne : [http://journals.openedition.org/carnets/1850] (consulté le 24 janvier 2019). 


\section{NOTES}

1. "L'exercice se distingue en effet de l'activité de résolution de problème par le souci de faire travailler l'élève sur l'acquisition d'une compétence restreinte portant sur une propriété particulière de la langue. »

2. Lépinette considère que « 'manuel' dénote un ouvrage dans lequel prédomine une organisation de type pédagogique » $(2012: 217)$.

3. Et sans doute plus tôt ailleurs ( $c f$. notamment les contributions de Marcus Reinfried et Norma Romanelli ainsi que le texte de présentation des éditeurs).

4. Nous excluons ici les ouvrages lexicographiques dont la nature ne se prête pas à l'inclusion d'exercices d'application.

5. Pour un panorama et une description détaillés de ces ouvrages $c f$. Viémon (2016a: 69-85).

6. Nous fournissons quelques explications sur ces exercices dans cette introduction; cependant nous invitons le lecteur à consulter Viémon (2016b) pour plus de précisions sur l'apparition des premiers exercices de prononciation dans les ouvrages grammaticaux de français en Espagne.

7. Les ouvrages s'intitulent respectivement Rudimentos breves y claros para aprender a leer, y escribir en francés sin socorro de maestro (1767) et Cartilla Francesa ó Combinaciones generales para la lectura (1781).

8. Contaut (1763) inclut également des prières - ainsi que des modèles de lettres - dans son ouvrage mais, contrairement à ceux de Soldevila et de Cañaveras, ce dernier est une grammaire non expressément destinée à l'apprentissage de la lecture/prononciation.

9. $C f$. note 5 .

10. Le «syllabaire » n'est pas ici un « livre d'apprentissage de la lecture à partir du découpage des mots en syllabes" (d'après le Trésor de la langue française) mais une série de combinaisons syllabiques organisées par type de syllabes (consonne + voyelle; voyelle + consonne ; consonne + voyelle + consonne ; etc.) et servant d'exercice de lecture à l'usager.

11. D'autres ouvrages que nous avons consultés, comme celui de Rovira (1835), par exemple, n'en proposent pas.

12. Ces textes sont mentionnés dans l'introduction de notre article.

13. En revanche, l'auteur applique sa méthode pour ce qui est de la morphologie : après chaque leçon, il propose un exercice d'application.

14. Cf. note 9 .

15. Par exemple, le $s$ simple représente le phonème /z/ et le double $s$ le phonème /s/; les voyelles nasales sont suivies d'un $n$ en petites capitales; / $\mathbb{E} /$ est représenté par le digramme eu en petites capitales également et surmonté d'une barre verticale, etc.

16. Nous n'avons malheureusement pas pu accéder aux leçons suivantes car l'édition que nous avons consultée est tronquée.

17. Par ailleurs, les exemples prennent une grande partie de l'espace car ils sont détachés des règles et imprimés en gras avec une taille de caractères bien supérieure à celle du texte théorique. Ce procédé typographique, qui démontre une conscience pédagogique certaine, est tout à fait inhabituel dans les ouvrages de ce type.

18. Pour plus de précisions sur cet auteur et son ouvrage, $c f$. Fernández Fraile (2002).

19. Nous sommes conscients du fait que Chantreau ne connaissait pas le concept moderne de phonème mais ces notations nous semblent les plus adéquates pour parler d'oppositions. 


\section{RÉSUMÉS}

Faisant suite à un travail précédent dans lequel nous étudiions l'apparition au XVIII ${ }^{\mathrm{e}}$ siècle des premiers exercices de prononciation du français destinés à un public hispanophone, le présent article traite de l'évolution de cette tendance dans la seconde moitié du XIX ${ }^{e}$ siècle; nous y analysons un corpus de vingt grammaires. Nous y constatons que : suivant un lent processus de manuélisation, de plus en plus d'auteurs introduisent des exercices d'application dans leur grammaire ; il existe une certaine continuité entre les deux époques, assurée entre autres par les nombreuses rééditions de Chantreau ; finalement certaines innovations voient le jour, que ce soit du point de vue du procédé utilisé dans la confection de l'exercice ou du point de vue de l'approche méthodologique.

Following a previous research work in which we studied the emergence in the eighteenth century of the first French pronunciation exercises aimed at a Spanish-speaking audience, the present article deals with the evolution of this trend in the second half of the nineteenth century, using a corpus of twenty grammar books. We observe that according to a slow transformation into textbooks, more and more authors include practical exercises in their grammars; that there is a certain continuity between the two periods which is guaranteed in part by the numerous reissues of Chantreau; finally, that some innovations emerge, whether we are considering the process used in designing the exercise, or the methodological approach.

\section{INDEX}

Mots-clés : histoire de la didactique des langues, exercice, prononciation du français, Espagne, XIXe siècle

Keywords : history of language learning and teaching (HoLLT), exercise, french pronunciation, Spain, nineteenth century

\section{AUTEUR}

\section{MARC VIÉMON}

Universidad de Sevilla

mviemon@us.es 